\title{
Prediction of the Active Ingredients and Mechanism of ASH against Liver Cancer Based on Network Pharmacology and Molecular Docking
}

\author{
Wenhua Guo',2, Kun Zhang'², Luhong Yang1,2* \\ ${ }^{1}$ Modern College of Humanities and Science of Shanxi Normal University, Linfen, China \\ ${ }^{2}$ School of Life Science, Shanxi Normal University, Linfen, China \\ Email: *ylh1010309@126.com
}

How to cite this paper: Guo, W.H., Zhang, K. and Yang, L.H. (2021) Prediction of the Active Ingredients and Mechanism of ASH against Liver Cancer Based on Network Pharmacology and Molecular Docking. Open Access Library Journal, 8: e7739. https://doi.org/10.4236/oalib.1107739

Received: July 8, 2021

Accepted: August 17, 2021

Published: August 20, 2021

Copyright $\odot 2021$ by author(s) and Open Access Library Inc.

This work is licensed under the Creative Commons Attribution International License (CC BY 4.0).

http://creativecommons.org/licenses/by/4.0/

\begin{abstract}
The network pharmacology and molecular docking technology were used to elucidate the mechanism of Artemisiae scopariae Herba (ASH) against liver cancer (LC). TCMSP and UniProt database were used to collect the active ingredients of ASH and predict their potential targets. The targets of LC were screened by GeneCards, OMIM and TTD database. The intersections of drug and disease targets were obtained by online software Venny 2.1, and the intersection targets were imported into R software (v3.6.3) for GO and KEGG function enrichment analysis. Construction of protein-protein interaction (PPI) network through STRING database, Cytoscape software was used to screen hub genes. Molecular docking analysis of hub genes was carried out with AutoDock vina software. A total of 13 active ingredients were screened out from ASH and 103 drug and disease intersection targets were screened. Finally, 7 hub targets including AKT1, TP53, JUN, MAPK1, TNF, RELA, IL6 were screened out. The hub targets were docked well with some active ingredients. The active ingredients of ASH are involved in hepatitis B, hepatitis C and other signaling pathways by acting on AKT1, TP53, JUN and other targets, which may play a role in the treatment of LC.
\end{abstract}

\section{Subject Areas}

Network Pharmacology

\section{Keywords}

Artemisiae scopariae Herba (ASH), Liver Cancer, Network Pharmacology, Molecular Docking 


\section{Introduction}

Artemisiae scopariae Herba (ASH) also called Artemisia capillaris (AC), belongs to the family of Asteraceae and the genus Artemisia [1]. As a therapeutic traditional medicine, it showed the anti-inflammatory effects in chronic hepatitis B virus infection and liver cirrhosis [2]. Moreover, the major constituents of ASH such as capillin and scoparone exhibit anti-cancer effects in liver, prostate, and lung cancers [2]. The extract of ASH (AC68) not only induced apoptosis but also inhibited cell growth, migration, and invasion of liver cancer cells by blocking the PI3K/AKT pathway [3]. ASH has certain effects on the treatment of hepatitis, also inhibits cell growth, invasion, and metastasis in liver cancer, together with induction of apoptosis, as well as hepatoprotective property [3] [4].

Liver cancer (LC) is the second most common cause of cancer-related death worldwide, it ranks fifth in terms of global cases and second in terms of deaths for males, hepatocellular carcinoma (HCC) is the most common type of LC worldwide [5] [6]. Lack of suitable biomarkers for early detection and limited treatment strategies are the major causes of high mortality [7]. A variety of risk factors have been associated with the development of LC, including hepatitis viruses, cirrhosis obesity and fatty liver disease [8]. The tumor microenvironment (TME) plays an important role in tumor progression and metastasis which contributes to tumor cell proliferation, survival, migration, and invasion [9]. More and more studies have revealed that TME has critical roles in the progression of LC [10].

There are several treatment options for LC including chemotherapy, surgery, radiation and immunotherapy [11]. Unfortunately, each of the treatment options suffers some drawbacks. Chinese medicines (CMs) have potential to both prevent LC occurrence and retard LC progression. The actions of CMs on LC may include tumor growth inhibition, antimetastatic activities, anti-inflammation, anti-LC stem cells, reversal on multi-drug resistance and induction/reduction of oxidative stress [12]. As a traditional Chinese medicine, ASH shows hepatoprotective property, and there is no effective therapy available to treat $\mathrm{LC}$ at present, which is expected to have a certain effect in the treatment of LC.

\section{Methods}

\subsection{The Putative Targets of ASH}

"Artemisiae scopariae Herba" was used as a keyword in the traditional Chinese medicine system pharmacology database and analysis platform (TCMSP, http://tcmspw.com/tcmsp.php) to get the ingredients of ASH and the parameters for the selection of active ingredients were set as follows: oral bioavailability $(\mathrm{OB}) \geq 30 \%$ and drug-likeness $(\mathrm{DL}) \geq 0.18$ [13]. In addition, potential targets of active ingredients were obtained from the TCMSP database, then the target proteins were imported into the UniProt database (https://www.uniprot.org/) to obtain the gene names. 


\subsection{Related Targets of LC and Prediction of Potential Targets of ASH against LC}

Liver cancer related genes were retrieved from Genecards

(https://www.genecards.org/), OMIM (https://omim.org/) and TTD

(http://db.idrblab.net/ttd/) database. The search results from each database were combined and duplicates were removed.

Online software Venny 2.1

(https://bioinfogp.cnb.csic.es/tools/venny/index.html) was used to obtain the common targets between ASH and LC. The "drug-ingredient-disease-target" network of ASH anti-LC was constructed by using Cytoscape software (v3.7.2).

\subsection{Functional Enrichment Analysis}

The common targets were used for GO and KEGG pathway enrichment with the Cluster Profiler package in R software (v3.6.3), and the "ggplot2" package was used to visualize the GO and KEGG enrichment results [14].

\subsection{Protein-Protein Interaction (PPI) Network Construction and Hub Genes Screening}

The PPI network was retrieved from STRING Version 11.0 (https://string-db.org/) by selecting Homo sapiens as the organism, and a confidence score $>0.9$ (highest confidence) was set as significant [15]. PPI network was then visualized by Cytoscape software (v3.7.2), CytoNCA plugin of Cytoscape was used to screen hub genes based on three criteria: degree centrality (DC), betweenness centrality (BC) and closeness centrality (CC) [16].

\subsection{Molecular Docking Simulation}

The molecular docking was performed to further investigate interactions between hub targets and their correspondent active ingredients. The structures of hub target proteins were obtained from the PDB database (https://www.rcsb.org/) and processed with PyMOL software. The 2D structure of the active ingredients was downloaded from PubChem (https://pubchem.ncbi.nlm.nih.gov) and energy minimised using MM2 in Chem3D. Afterwards, both the ligand and the receptor were converted to the PDBQT format using AutoDock Tools (ADT 1.5.6), and the docking is performed by running Vina. If the binding energy is less than -5 $\mathrm{kJ} \cdot \mathrm{mol}^{-1}$, it indicated that the target has certain binding activity with the ingredient, the lower the binding energy value, the stronger the binding to the target protein [17].

\section{Results}

\subsection{Active Compounds and Targets of ASH}

A total of 13 active ingredients of ASH were screened from TCMSP database, the correspondent target proteins of each active ingredient were also obtained (Table 1). After removing duplicated targets, we obtained 169 standard gene 
names (based on UniProt annotation).

\subsection{Gene Targets of LC and Potential Targets of ASH against LC}

A total of 1004 targets for LC were collected from the GeneCards database by setting relevance score $>20$, we identify 493 and 13 LC-related targets from the OMIM and TTD databases. After merging and deleting the duplicate genes, we collected 1410 LC-related target genes.

Venn diagram was generated through the online tool Venny 2.1 and acquired 103 genes about ASH against LC (Figure 1). To further discover the mechanism of ASH against LC, the PPI network of targets for ASH anti-LC was shown as Figure 2.

Table 1. Active ingredients in Artemisiae scopariae Herba (ASH).

\begin{tabular}{cccc}
\hline Mol ID & Molecule Name & OB (\%) & DL \\
MOL008045 & 4'-Methylcapillarisin & 72.18 & 0.35 \\
MOL008047 & Artepillin A & 68.32 & 0.24 \\
MOL008043 & capillarisin & 57.56 & 0.31 \\
MOL008039 & Isoarcapillin & 57.4 & 0.41 \\
MOL008046 & Demethoxycapillarisin & 52.33 & 0.25 \\
MOL000354 & isorhamnetin & 49.6 & 0.31 \\
MOL004609 & Areapillin & 48.96 & 0.41 \\
MOL000098 & quercetin & 46.43 & 0.28 \\
MOL008040 & Eupalitin & 46.11 & 0.33 \\
MOL008041 & Eupatolitin & 42.55 & 0.37 \\
MOL005573 & Genkwanin & 37.13 & 0.24 \\
MOL000358 & beta-sitosterol & 36.91 & 0.75 \\
MOL007274 & Skrofulein & 30.35 & 0.3 \\
\hline
\end{tabular}

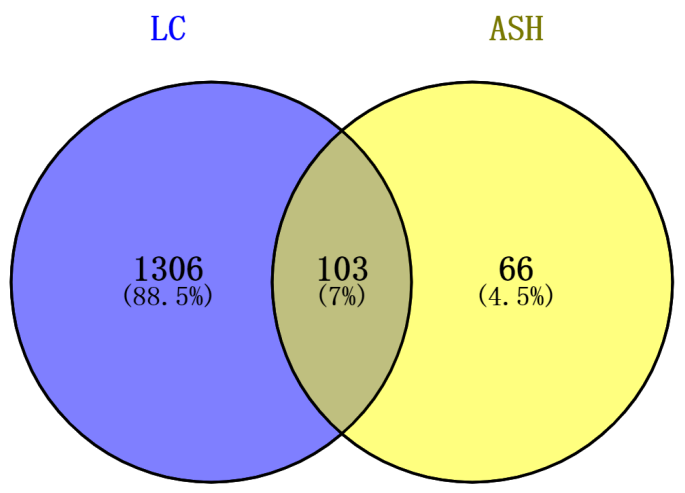

Figure 1. Venn diagram of the targets in LC and ASH. Blue indicates LC targets, yellow indicates ASH targets, the intersection in yellow shaded indicates the common targets between LC and ASH. 


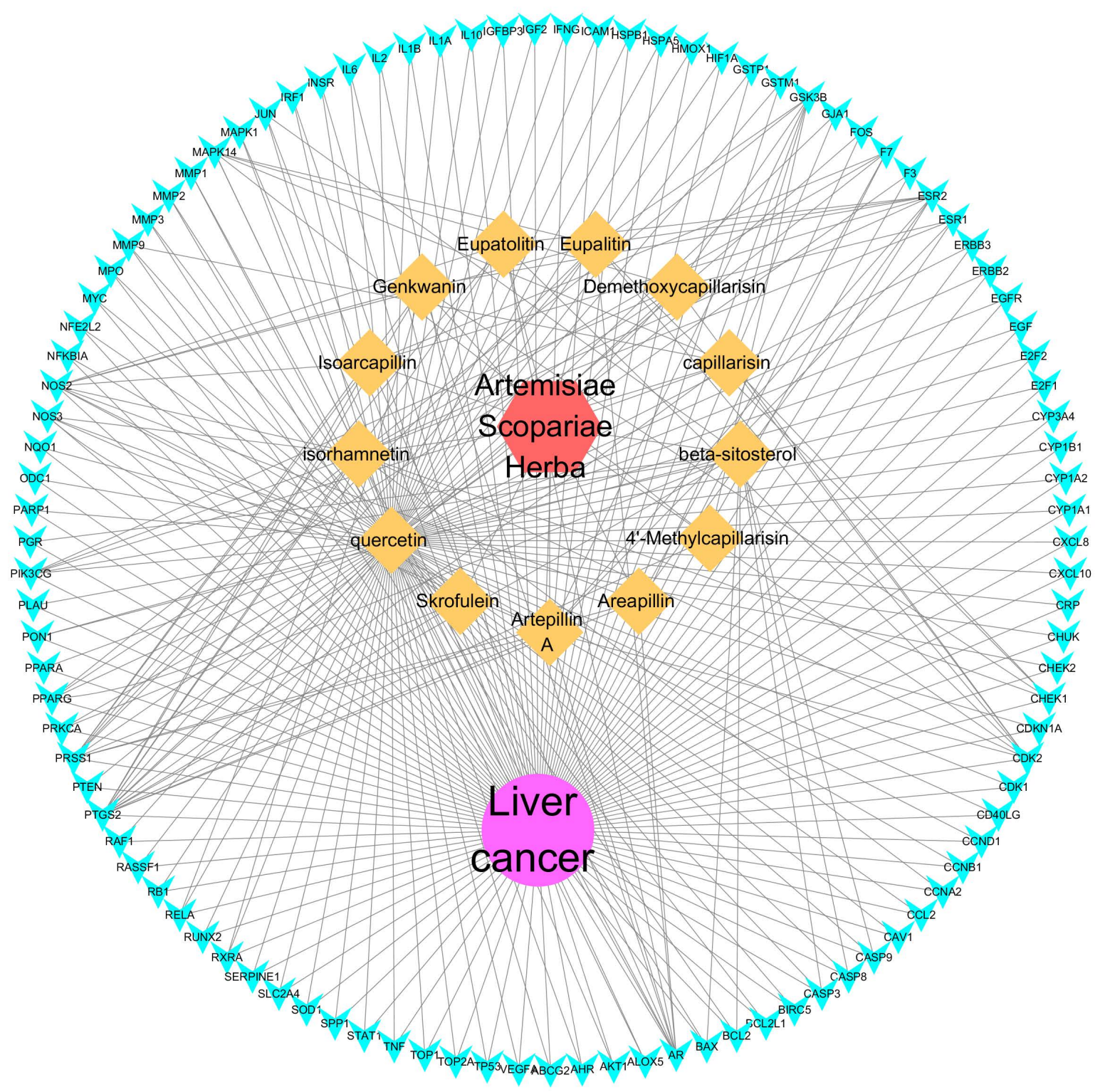

Figure 2. PPI network of targets for ASH against LC (yellow diamonds represent 13 active ingredients in ASH; blue nodes represent intersection targets between ASH and LC targets).

\subsection{Enrichment Analysis}

1775 GO terms were obtained with p.adjust $<0.01$, there are 1665 terms of biological process (BP), 24 terms of cell composition (CC) and 86 terms of molecular function (MF), accounting for $93.8 \%, 1.4 \%$ and $4.8 \%$ respectively. Then the top 5 BP, CC, MF terms are visualized (Table 2, Figure 3).

In total, 131 KEGG pathways were significantly enriched (p.adjust $<0.01$ ), and the top 10 were visualized (Table 3, Figure 4), involved in the prostate cancer, hepatitis B, bladder cancer, kaposi sarcoma-associated herpesvirus infection, hepatitis C, AGE-RAGE signaling pathway in diabetic complications, small cell lung cancer, fluid shear stress and atherosclerosis, IL-17 signaling pathway, human cytomegalovirus infection. 
Table 2. Gene Ontology (GO) enrichment.

\begin{tabular}{|c|c|c|c|c|}
\hline Ontology & ID & Description & p.adjust & Count \\
\hline BP & GO:0000302 & response to reactive oxygen species & $6.19456 \mathrm{E}-25$ & 27 \\
\hline $\mathrm{BP}$ & GO:0034599 & cellular response to oxidative stress & $6.64222 \mathrm{E}-25$ & 29 \\
\hline $\mathrm{BP}$ & GO:0032496 & response to lipopolysaccharide & $5.39618 \mathrm{E}-24$ & 29 \\
\hline BP & GO:0006979 & response to oxidative stress & $5.39618 \mathrm{E}-24$ & 32 \\
\hline $\mathrm{BP}$ & GO:0002237 & response to molecule of bacterial origin & $1.07768 \mathrm{E}-23$ & 29 \\
\hline MF & GO:0044389 & ubiquitin-like protein ligase binding & $1.00158 \mathrm{E}-08$ & 16 \\
\hline MF & GO:0005126 & cytokine receptor binding & $1.92422 \mathrm{E}-08$ & 15 \\
\hline MF & GO:0004879 & nuclear receptor activity & $2.36334 \mathrm{E}-08$ & 8 \\
\hline MF & GO:0098531 & $\begin{array}{l}\text { transcription factor activity, direct ligand } \\
\text { regulated sequence-specific DNA binding }\end{array}$ & $2.36334 \mathrm{E}-08$ & 8 \\
\hline MF & GO:0005125 & cytokine activity & $3.44479 \mathrm{E}-08$ & 13 \\
\hline $\mathrm{CC}$ & GO:0005667 & transcription factor complex & $1.72032 \mathrm{E}-07$ & 15 \\
\hline $\mathrm{CC}$ & GO:0000307 & $\begin{array}{l}\text { cyclin-dependent protein kinase } \\
\text { holoenzyme complex }\end{array}$ & $2.39584 \mathrm{E}-07$ & 7 \\
\hline $\mathrm{CC}$ & GO:1902911 & protein kinase complex & $4.47512 \mathrm{E}-07$ & 9 \\
\hline $\mathrm{CC}$ & GO:0045121 & membrane raft & $5.05468 \mathrm{E}-07$ & 13 \\
\hline $\mathrm{CC}$ & GO:0098857 & membrane microdomain & $5.05468 \mathrm{E}-07$ & 13 \\
\hline
\end{tabular}

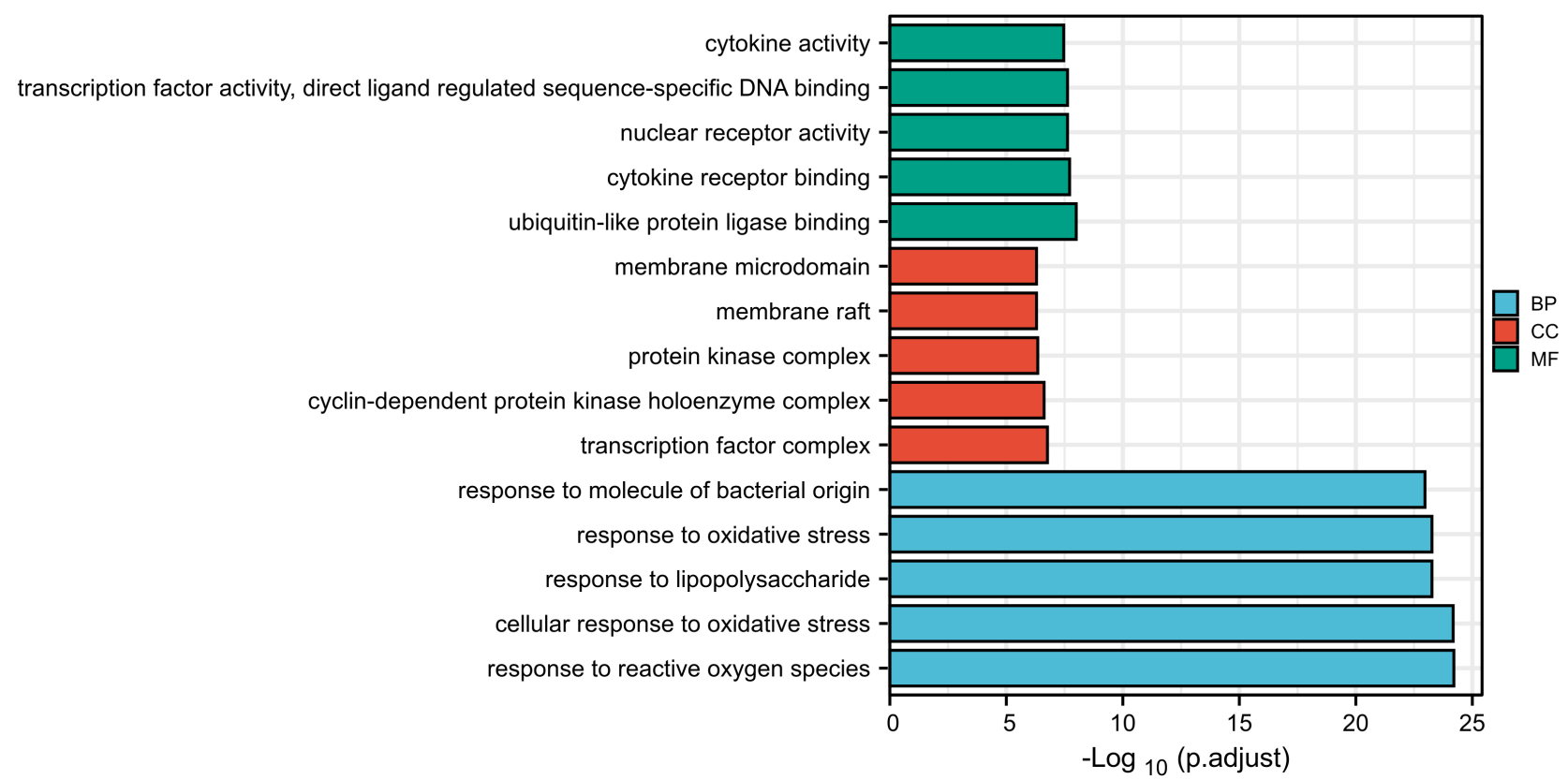

Figure 3. GO enrichment analysis (adjusted p value < 0.01). The y-axis represents GO categories identified in the GO analysis, and the $\mathrm{x}$-axis represents the significance $\left[-\log _{10}\right.$ (p.adjust)]. Blue, red, and green boxes represent GO BP, CC, and MF, respectively. 


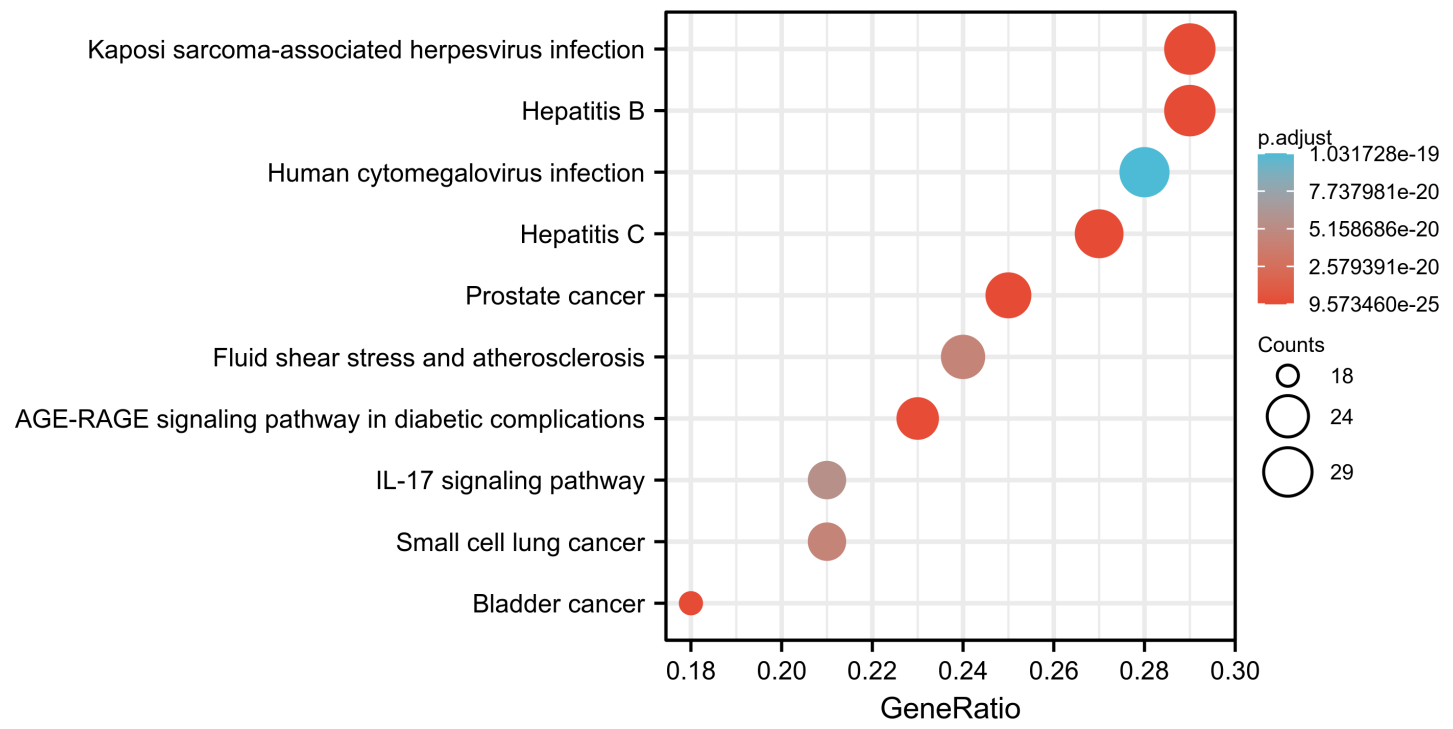

Figure 4. KEGG pathway enrichment analysis (adjusted $\mathrm{p}$ value $<0.01$ ). The $\mathrm{y}$-axis shows the pathway term, and the $\mathrm{x}$-axis shows the Gene Ratio of each KEGG pathway. The color indicates the significance $\left[-\log _{10}\right.$ (p.adjust)], and the size of the circle represents the number of genes enriched in the corresponding pathways.

Table 3. Kyoto Encyclopedia of Genes and Genomes (KEGG) enrichment.

\begin{tabular}{|c|c|c|c|}
\hline ID & Description & p.adjust & Count \\
\hline hsa05215 & Prostate cancer & $9.57346 \mathrm{E}-25$ & 25 \\
\hline hsa05161 & Hepatitis B & $1.86759 \mathrm{E}-24$ & 29 \\
\hline hsa05219 & Bladder cancer & $1.12109 \mathrm{E}-22$ & 18 \\
\hline hsa05167 & $\begin{array}{l}\text { Kaposi sarcoma-associated } \\
\text { herpesvirus infection }\end{array}$ & $1.63617 \mathrm{E}-22$ & 29 \\
\hline hsa05160 & Hepatitis C & $1.63617 \mathrm{E}-22$ & 27 \\
\hline hsa04933 & $\begin{array}{l}\text { AGE-RAGE signaling pathway in } \\
\text { diabetic complications }\end{array}$ & $4.08257 \mathrm{E}-22$ & 23 \\
\hline hsa05222 & Small cell lung cancer & $4.42098 \mathrm{E}-20$ & 21 \\
\hline hsa05418 & Fluid shear stress and atherosclerosis & $4.43383 \mathrm{E}-20$ & 24 \\
\hline hsa04657 & IL-17 signaling pathway & $5.61179 \mathrm{E}-20$ & 21 \\
\hline hsa05163 & Human cytomegalovirus infection & $1.03173 \mathrm{E}-19$ & 28 \\
\hline
\end{tabular}

\subsection{PPI Network and Hub Genes}

The PPI network was constructed by STRING at a confidence value of 0.9 and it included 100 nodes and 434 edges (Figure 5, Table 4). Nodes which had high degree were identified as the hub nodes in the PPI network, hub genes with degree $\geq 20$ were selected (AKT1, TP53, JUN, MAPK1, TNF, RELA, IL6). 


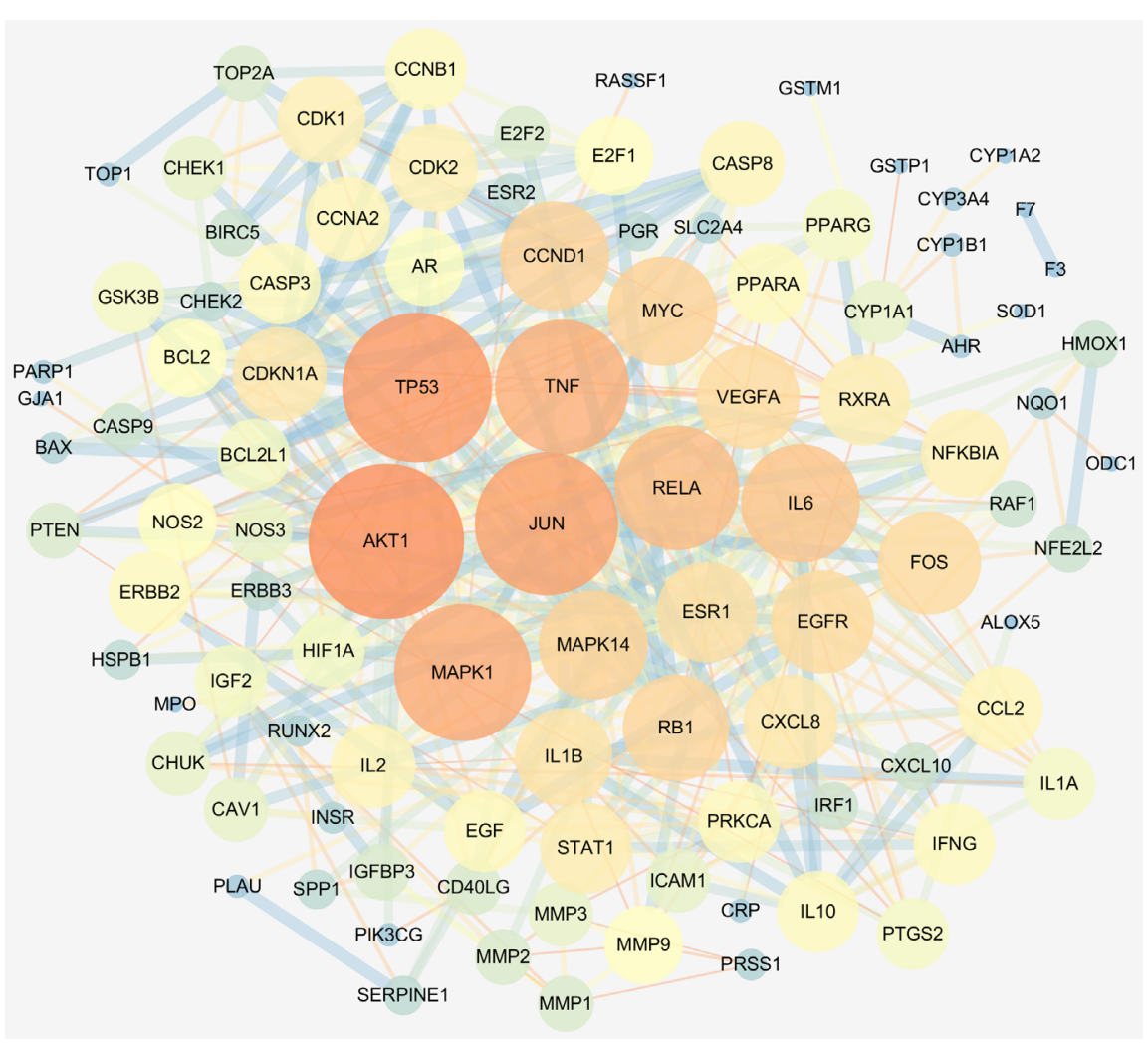

Figure 5. Protein-protein interaction (PPI) network of common genes. The nodes represent genes, and the size and color represent the degree value (the higher the degree, the larger the node and the brighter of the color). The thickness and color of the edges display the combined-score between two genes, the higher the score, the wider the edge and the darker of the color.

Table 4. Topological information of 7 hub targets.

\begin{tabular}{cccc}
\hline Gene & DC & BC & CC \\
\hline AKT1 & 34 & 1480.8927 & 0.26052633 \\
TP53 & 32 & 1257.4023 & 0.2578125 \\
JUN & 30 & 932.47144 & 0.26052633 \\
MAPK1 & 28 & 1087.2823 & 0.25647667 \\
TNF & 27 & 659.35925 & 0.25 \\
RELA & 24 & 334.47003 & 0.25 \\
IL6 & 22 & 522.2917 & 0.24029127 \\
\hline
\end{tabular}

\subsection{Molecular Docking}

Molecular docking results showed that hub protein targets and active ingredients showed good binding interactions (Table 5). Among them, the compound quercetin and MAPK1 displayed the lowest binding energy (-8.9), which suggests that quercetin demonstrated the best docking score against MAPK1 (Figure 6). 
Table 5. The binding energy of the hub targets bound to the active ingredients.

\begin{tabular}{ccc}
\hline Target & Ingredient & Binding Energy $/ \mathrm{kJ} \cdot \mathrm{mol}^{-1}$ \\
AKT1 & quercetin & -6.9 \\
TP53 & quercetin & -7.2 \\
JUN & beta-sitosterol & -7.9 \\
& quercetin & -8.8 \\
MAPK1 & quercetin & -8.9 \\
TNF & quercetin & -5.2 \\
RELA & isorhamnetin & -7.1 \\
& quercetin & -7.7 \\
IL6 & quercetin & -7.6 \\
\hline
\end{tabular}

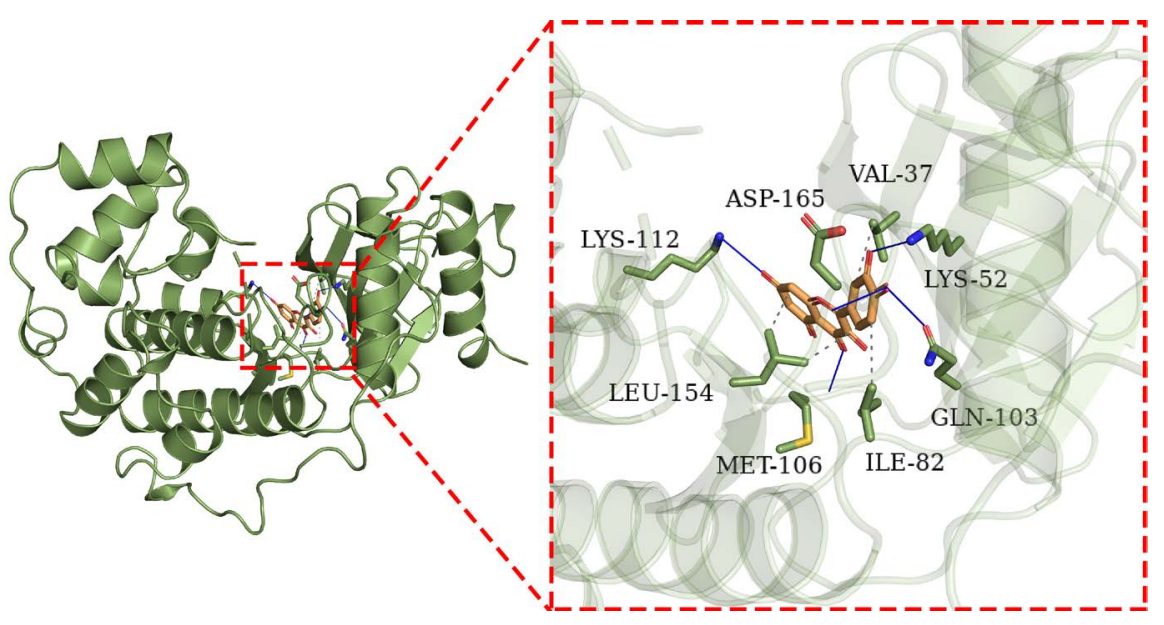

Figure 6. MAPK1-quercetin docking. Quercetin forms 4 hydrophobic interactions with VAL37, ILE82, LEU154, forms 5 H-bonds with LYS52, GLN103, MET106, LYS112, ASP165.

\section{Discussion}

In this study, a total of 13 active ingredients were obtained from Artemisiae scopariae Herba, including flavonoids, chromones and phytosterols. Flavonoids are well known for their physiological anti-inflammatory and antitumor activities, flavonoids may modulate almost all key processes involved in carcinogenesis including apoptosis, proliferation, angiogenesis and metastatic progression [18] [19]. In recent years, various flavonoids have been recognized as having potential protective activity against artificially induced-liver damage [20]. Chromones have been reported to possess antimicrobial, antiviral, and antitumoral activities and the ability to inhibit several enzymes, it also affects the function and activity of liver-metabolizing enzymes [21]. Phytosterols possess hepatoprotective effect, and the anti-cancer effect of phytosterols are achieved by inhibition of cell cycle progression, promotion of cellular apoptosis, inhibition of cell 
invasion, migration and adhesion, as well as stimulation of the immune function [22].

The PPI network showed that the targets of ASH against LC do not work alone but instead is a complex interconnected network, according to degree value we finally screened out 7 hub genes (AKT1, TP53, JUN, MAPK1, TNF, RELA, IL6). AKT1 belongs to the family of serine/threonine protein kinases (AKT1, AKT2, and AKT3) known as AKT kinases, AKT is closely associated with cell survival, proliferation, apoptosis, migration and angiogenesis in hepatocellular carcinoma (HCC) [23] [24]. AKT1 participates in the initiation, progression and metastasis of malignant tumors, silencing AKT1 significantly stimulated apoptosis and suppressed the cell cycle, whereas increasing AKT1 expression promoted HCC cells proliferation [24].

TP53 is the most widely studied tumor suppressor gene, playing an important role in inhibiting tumor development, the function of it is to inhibit cell proliferation in response to DNA damage. By regulating target genes, TP53 induces a variety of cellular responses, including growth arrest, senescence, and apoptosis [25]. JUN encodes c-Jun protein which has essential influence in cell proliferation, survival, and death [26] [27]. Hepatitis C virus infection stimulates c-Jun signaling via protein kinase R to promote proliferation of HCC [27].

MAPK1 plays a votal role in cancer progression, especially in cancer metastasis, and in HCC development, simultaneous activation of the MAPK1 pathways has been shown to enhance cell-cycle progression [28]. Tumor necrosis factor (TNF) is a mediator of the acute phase response in the liver and can initiate proliferation and cause cell death in hepatocytes, participates in many forms of hepatic pathology, including ischemia/reperfusion injury, alcoholic and viral hepatitis, and injury by hepatotoxins [29] [30]. Study has shown that TNF-a expression in HCC is significantly higher than that in normal hepatic tissue, positively related with the proliferation and invasion ability of HCC cells [31].

RELA, a member of the NF- $\kappa B$ family, work as a potential factor in the onset and progression of cancers through regulating the expression of genes linked to cell proliferation, migration, invasion, etc. [32] [33]. Clinically, RelA expression has been associated with a lower degree of apoptosis and cirrhosis in the livers of patients with hepatitis C, and liver RelA mRNA levels were inversely associated with severe liver damage and mortality [34]. IL-6 is a cytokine produced by various cells serve a key function in the proliferation, apoptosis, recurrence and metastasis of liver cancer cells [35]. High IL6 level linked with patients' mortality in cirrhotic patients caused by hepatitis B virus and hepatitis $C$ virus infection. Similarly, high serum level of IL6 was associated with liver-related mortality in chronic HCV patients [36].

\section{Conclusion}

In this study, we adopted network pharmacology and molecular docking technology to explore the mechanism of ASH anti-LC. The results indicated that 
ASH may interact with hub genes such as AKT1, TP53, JUN, etc., regulates hepatitis $B$, hepatitis $C$ and other signaling pathways, which exerts anticancer effects. Although we lacked experimental validation, it also provides theoretical basis for the treatment of LC in the future.

\section{Acknowledgements}

The study was supported by the Basic Research Foundation of School of Modern College of Humanities and Sciences of Shanxi Normal University (Grant No. 2020JCYJ19).

\section{Conflicts of Interest}

The authors declare no conflicts of interest.

\section{References}

[1] Son, H.U., Lee, S., Heo, J.C. and Lee, S.H. (2017) The Solid-State Fermentation of Artemisia capillaris Leaves with Ganoderma lucidum Enhances the Anti-Inflammatory Effects in a Model of Atopic Dermatitis. International Journal of Molecular Medicine, 39, 1233-1241. https://doi.org/10.3892/ijmm.2017.2945

[2] Kim, J., Jung, K.H., Yan, H.H., Cheon, M.J., Kang, S., et al. (2018) Artemisia capillaris Leaves Inhibit Cell Proliferation and Induce Apoptosis in Hepatocellular Carcinoma. BMC Complementary and Alternative Medicine, 18, 147. https://doi.org/10.1186/s12906-018-2217-6

[3] Yan, H., Jung, K.H., Kim, J., Rumman, M., Oh, M.S., et al. (2018) Artemisia capillaris Extract AC68 Induces Apoptosis of Hepatocellular Carcinoma by Blocking the PI3K/AKT Pathway. Biomedicine \& Pharmacotherapy, 98, 134-141. https://doi.org/10.1016/j.biopha.2017.12.043

[4] Jang, E., Kim, B.J., Lee, K.T., Inn, K.S. and Lee, J.H. (2015) A Survey of Therapeutic Effects of Artemisia capillaris in Liver Diseases. Evidence-Based Complementary and Alternative Medicine, 2015, Article ID: 728137. https://doi.org/10.1155/2015/728137

[5] Tseng, H.C., Xiong, W., Badeti, S., Yang, Y., Ma, M., et al. (2020) Efficacy of Anti-CD147 Chimeric Antigen Receptors Targeting Hepatocellular Carcinoma. Nature Communications, 11, 4810. https://doi.org/10.1038/s41467-020-18444-2

[6] Yapasert,R., Lertprasertsuk, N., Subhawa, S., Poofery, J., Sripanidkulchai, B., et al. (2020) Antitumor Efficacy of the Herbal Recipe Benja Amarit against Highly Invasive Cholangiocarcinoma by Inducing Apoptosis Both in Vitro and in Vivo. International Journal of Molecular Sciences, 21, 5669. https://doi.org/10.3390/ijms21165669

[7] Liu, M., Yan, Q., Sun, Y., Nam, Y., Hu, L., et al. (2020) A Hepatocyte Differentiation Model Reveals Two Subtypes of Liver Cancer with Different Oncofetal Properties and Therapeutic Targets. Proceedings of the National Academy of Sciences of the United States of America, 117, 6103-6113. https://doi.org/10.1073/pnas.1912146117

[8] Anwanwan, D., Singh, S.K., Singh, S., Saikam, V. and Singh, R. (2020) Challenges in Liver Cancer and Possible Treatment Approaches. Biochimica et Biophysica Acta. Reviews on Cancer, 1873, Article ID: 188314. https://doi.org/10.1016/j.bbcan.2019.188314

[9] Wang, X., Hu, Z., Wang, Z., Cui, Y. and Cui, X. (2019) Angiopoietin-Like Protein 2 
Is an Important Facilitator of Tumor Proliferation, Metastasis, Angiogenesis and Glycolysis in Osteosarcoma. American Journal of Translational Research, 11, 6341-6355.

[10] Tian, X., Wu, Y., Yang, Y., Wang, J., Niu, M., et al. (2020) Long Noncoding RNA LINC00662 Promotes M2 Macrophage Polarization and Hepatocellular Carcinoma Progression via Activating Wnt/ $\beta$-Catenin Signaling. Molecular Oncology, 14, 462-483. https://doi.org/10.1002/1878-0261.12606

[11] Hefnawy, A., Khalil, I.H., Arafa, K., Emara, M. and El-Sherbiny, I.M. (2020) Dual-Ligand Functionalized Core-Shell Chitosan-Based Nanocarrier for Hepatocellular Carcinoma-Targeted Drug Delivery. International Journal of Nanomedicine, 15, 821-837. https://doi.org/10.2147/IJN.S240359

[12] Wang, X., Wang, N., Cheung, F., Lao, L., Li, C., et al. (2015) Chinese Medicines for Prevention and Treatment of Human Hepatocellular Carcinoma: Current Progress on Pharmacological Actions and Mechanisms. Journal of Integrative Medicine, 13, 142-164. https://doi.org/10.1016/S2095-4964(15)60171-6

[13] Jian, G.H., Su, B.Z., Zhou, W.J. and Xiong, H. (2020) Application of Network Pharmacology and Molecular Docking to Elucidate the Potential Mechanism of Eucommia ulmoides-Radix Achyranthis bidentatae against Osteoarthritis. BioData Mining, 13, 12. https://doi.org/10.1186/s13040-020-00221-y

[14] Zhang, Y., Tseng, J.T., Lien, I.C., Li, F., Wu, W., et al. (2020) mRNAsi Index: Machine Learning in Mining Lung Adenocarcinoma Stem Cell Biomarkers. Genes, 11, 257. https://doi.org/10.3390/genes11030257

[15] Fang, J., Pan, Z., Yu, H., Yang, S., Hu, X., et al. (2020) Regulatory Master Genes Identification and Drug Repositioning by Integrative mRNA-miRNA Network Analysis for Acute Type A Aortic Dissection. Frontiers in Pharmacology, 11, Article ID: 575765. https://doi.org/10.3389/fphar.2020.575765

[16] Cao, F., Guo, Y., Zhang, Q., Fan, Y., Liu, Q., et al. (2020) Integration of Transcriptome Resequencing and Quantitative Proteomics Analyses of Collagenase VII-Induced Intracerebral Hemorrhage in Mice. Frontiers in Genetics, 11, Article ID: 551065. https://doi.org/10.3389/fgene.2020.551065

[17] Yin, B., Bi, Y.M., Fan, G.J. and Xia, Y.Q. (2020) Molecular Mechanism of the Effect of Huanglian Jiedu Decoction on Type 2 Diabetes Mellitus Based on Network Pharmacology and Molecular Docking. Journal of Diabetes Research, 2020, Article ID: 5273914. https://doi.org/10.1155/2020/5273914

[18] Samec, M., Liskova, A., Koklesova, L., Samuel, S.M., Zhai, K., et al. (2020) Flavonoids against the Warburg Phenotype-Concepts of Predictive, Preventive and Personalised Medicine to Cut the Gordian Knot of Cancer Cell Metabolism. The EPMA Journal, 11, 377-398. https://doi.org/10.1007/s13167-020-00217-y

[19] Liu, X., Hu, P., Li, H., Yu, X.X., Wang, X.Y., et al. (2020) LW-213, a Newly Synthesized Flavonoid, Induces G2/M Phase Arrest and Apoptosis in Chronic Myeloid Leukemia. Acta Pharmacologica Sinica, 41, 249-259.

https://doi.org/10.1038/s41401-019-0270-4

[20] Liao, C.Y., Lee, C.C., Tsai, C.C., Hsueh, C.W., Wang, C.C., et al. (2015) Novel Investigations of Flavonoids as Chemopreventive Agents for Hepatocellular Carcinoma. BioMed Research International, 2015, Article ID: 840542. https://doi.org/10.1155/2015/840542

[21] Silva, C.F.M., Batista, V.F., Pinto, D. and Silva, A.M.S. (2018) Challenges with Chromone as a Privileged Scaffold in Drug Discovery. Expert Opinion on Drug Discovery, 13, 795-798. https://doi.org/10.1080/17460441.2018.1494720 
[22] Shahzad, N., Khan, W., Md, S., Ali, A., Saluja, S.S., et al. (2017) Phytosterols as a Natural Anticancer Agent: Current Status and Future Perspective. Biomedicine \& Pharmacotherapy, 88, 786-794. https://doi.org/10.1016/j.biopha.2017.01.068

[23] Gu, Z., Wang, L., Yao, X., Long, Q., Lee, K., et al. (2020) ClC-3/SGK1 Regulatory Axis Enhances the Olaparib-Induced Antitumor Effect in Human Stomach Adenocarcinoma. Cell Death \& Disease, 11, 898. https://doi.org/10.1038/s41419-020-03107-3

[24] Chen, J., Liang, J., Liu, S., Song, S., Guo, W., et al. (2018) Differential Regulation of AKT1 Contributes to Survival and Proliferation in Hepatocellular Carcinoma Cells by Mediating Notch1 Expression. Oncology Letters, 15, 6857-6864. https://doi.org/10.3892/ol.2018.8193

[25] Yu, Y., Gao, C., Chen, Y., Wang, M., Zhang, J., et al. (2020) Copy Number Analysis Reveal Genetic Risks of Penile Cancer. Frontiers in Oncology, 10, Article ID: 596261. https://doi.org/10.3389/fonc.2020.596261

[26] Geng, X., Wang, Y., Hong, Q., Yang, J., Zheng, W., et al. (2015) Differences in Gene Expression Profiles and Signaling Pathways in Rhabdomyolysis-Induced Acute Kidney Injury. International Journal of Clinical and Experimental Pathology, 8, 14087-14098.

[27] Chang, L., Yuan, W. and Zhu, L. (2020) $\beta$-Cantenin Is Potentially Involved in the Regulation of c-Jun Signaling Following Bovine Herpesvirus 1 Infection. Veterinary Microbiology, 248, Article ID: 108804. https://doi.org/10.1016/j.vetmic.2020.108804

[28] Jiang, G., Zhang, L., Zhu, Q., Bai, D., Zhang, C., et al. (2016) CD146 Promotes Metastasis and Predicts Poor Prognosis of Hepatocellular Carcinoma. Journal of Experimental \& Clinical Cancer Research, 35, 38. https://doi.org/10.1186/s13046-016-0313-3

[29] Roderburg, C., Gautheron, J. and Luedde, T. (2012) TNF-Dependent Signaling Pathways in Liver Cancer: Promising Targets for Therapeutic Strategies? Digestive Diseases (Basel, Switzerland), 30, 500-507. https://doi.org/10.1159/000341700

[30] Pierce, R.H., Campbell, J.S., Stephenson, A.B., Franklin, C.C., Chaisson, M., et al. (2000) Disruption of Redox Homeostasis in Tumor Necrosis Factor-Induced Apoptosis in a Murine Hepatocyte Cell Line. The American Journal of Pathology, 157, 221-236. https://doi.org/10.1016/S0002-9440(10)64533-6

[31] Tan, W., Luo, X., Li, W., Zhong, J., Cao, J., et al. (2019) TNF- $\alpha$ Is a Potential Therapeutic Target to Overcome Sorafenib Resistance in Hepatocellular Carcinoma. EBioMedicine, 40, 446-456. https://doi.org/10.1016/j.ebiom.2018.12.047

[32] Luo, Z., Yi, Z.J., Ou, Z.L., Han, T., Wan, T., et al. (2019) RELA/NEAT1/ miR-302a-3p/RELA Feedback Loop Modulates Pancreatic Ductal Adenocarcinoma Cell Proliferation and Migration. Journal of Cellular Physiology, 234, 3583-3597. https://doi.org/10.1002/jcp.27039

[33] Ricca, A., Biroccio, A., Trisciuoglio, D., Cippitelli, M., Zupi, G., et al. (2001) relA Over-Expression Reduces Tumorigenicity and Activates Apoptosis in Human Cancer Cells. British Journal of Cancer, 85, 1914-1921.

https://doi.org/10.1054/bjoc.2001.2174

[34] Kim, Y., Allen, E., Baird, L.A., Symer, E.M., Korkmaz, F.T., et al. (2019) NF- $\kappa$ B Re1A Is Required for Hepatoprotection during Pneumonia and Sepsis. Infection and Immunity, 87, e00132-19. https://doi.org/10.1128/IAI.00132-19

[35] Chen, S., Tang, Y., Yang, C., Li, K., Huang, X., et al. (2020) Silencing CDC25A Inhibits the Proliferation of Liver Cancer Cells by Downregulating IL-6 in Vitro and in Vivo. International Journal of Molecular Medicine, 45, 743-752. 
https://doi.org/10.3892/ijmm.2020.4461

[36] Song, Y., Yang, X., Shen, Y., Wang, Y., Xia, X., et al. (2019) STAT3 Signaling Pathway Plays Importantly Genetic and Functional Roles in HCV Infection. Molecular Genetics \& Genomic Medicine, 7, e821. https://doi.org/10.1002/mgg3.821 